\title{
NGHIÊN CÚ̉U KHẢ NĂNG PHÁT HIỆN BỤI PM10 TRONG KHÔNG KHÍ BÀ̀NG DŨ LIỆ ẢNH VỆ TINH LANDSAT 8 OLI KHU VỰC HÀ NỘI
}

\author{
NGUYẼN NHƯ' HÙNG ${ }^{(1)}$, TRẦ VÂN ANH(2) \\ ${ }^{(1)} H o ̣ c$ viện Kỹ thuật Quân sụp \\ ${ }^{(2)} Đ a ̣ i$ học Mỏ - Địa chất Hà Nội
}

\section{Tóm tắt:}

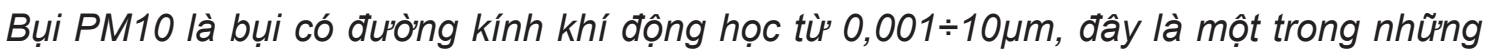
tác nhân ô nhiễm không khí ảnh hướng lớn tới sức khỏe con người. Trong bài báo này, chúng tôi tiến hành nghiên cứu xác định bưi PM10 trong không khí bằng cách sử dụng các hình ảnh vệ tinh Landsat $8 \mathrm{OLI}$ khu vực trung tâm thành phố Hà Nội. Trọng tâm nghiên cứu về khảo nghiệm quá trình xác định ô nhiễm không khí, bắt đầu từ việc điều chỉnh khí quyển của vệ tinh Landsat $8 \mathrm{OLI}$ bằng phương pháp DOS, tính $A O T$, hàm liên hệ giữa $A O T$ và PM10, từ đó tiến hành tính PM10 từ dữ liệu ảnh vệ tinh Landsat 8 OLI ở khu vực trung tâm của thành phố Hà Nội.

\section{1. Đặt vấn đề}

Ô nhiễm không khí là một mối quan tâm của nhiều nước trên thế giới. Việt Nam là một nước đang phát triển, quá trình đô thị hóa rất nhanh, việc xây dựng liên tục tăng, sự tăng trưởng của các ngành công nghiệp, tăng phương tiện giao thông, do đó ô nhiễm trong không khí ngày càng trở nên nghiêm trọng hơn. Theo Tố chức $\mathrm{Y}$ tế Thế giới (WHO), ô nhiễm không khí đô thị làm 800.000 người chết và 4,6 triệu giảm tuổi thọ trên thế giới mỗi năm, $2 / 3$ các ca tử vong và giảm tuổi thọ do ô nhiễm không khí của các nước đang phát triển ở châu Á.

Tuy nhiên, trong lĩnh vực giám sát ô nhiễm không khí, hiện nay ở Việt Nam chủ yếu là nội suy dựa trên dữ liệu từ các trạm giám sát, để đo lường và phủ trùm một khu vực có chi phí rất lớn. Vì vậy, việc cung cấp thông tin ô nhiễm trên một khu vực có diện tích lớn là rất khó khăn.

Các nghiên cứu trên thế giới đã chỉ ra rằng việc sử dụng các hình ảnh vệ tinh đa phổ hoàn toàn có thể phát hiện ô nhiễm không khí ở các khu vực mà chúng ta quan tâm. Một số nghiên cứu đã chỉ ra các mối quan hệ có thể có giữa các dữ liệu vệ tinh và ô nhiễm không khí như [10,11-12]. Có những nghiên cứu sử dụng dữ liệu vệ tinh khác nhau cho việc nghiên cứu khí quyển môi trường như: Sử dụng ảnh NOAA-14 AVHRR [1]; ảnh Landsat TM [7,8-11]; ảnh SPOT [9] và ảnh MODIS [13].

Ở Việt Nam, có một số tác giả đã nghiên cứu khả năng giám sát ô nhiễm không khí bằng cách sử dụng dữ liệu ảnh vệ tinh Landsat 7 [16,17]; sử dụng ảnh SPOT5 $[14,15]$

Hiện nay ảnh Landsat $8 \mathrm{OLI}$ đã đi vào hoạt động từ năm 2013, tuy nhiên việc nghiên cứu khả năng ứng dụng nó vào xác định bụi PM10 là chưa có. Vì vậy, trong nghiên cứu này, chúng tôi trình bày các tiềm năng thu hồi nồng độ các hạt vật chất có đường kính ít hơn mười micromet (PM10) trong khí quyển bằng cách sử dụng các hình ảnh vệ tinh Landsat 8 OLI thực nghiệm tại khu vực trung tâm thành phố Hà Nội. 


\section{Khu vực nghiên cứu}

Theo số liệu thống kê của công ty ARIA Technologies (công ty chuyên cung cấp giải pháp phần mềm tính toán, mô phỏng ô nhiễm môi trường không khí và hỗ trợ dự báo khí tượng) của Pháp cho thấy, mỗi năm Hà Nội có tốc độ tăng bình quân các phương tiện giao thông từ $12 \%-15 \%$, các phương tiện này góp phần lớn vào lượng phát thải độc hại như $\mathrm{SO}_{2}, \mathrm{NO}_{\mathrm{x}}$. Chuyên gia Jacques Moussafir, công ty ARIA Technologies cảnh báo "Tại các đô thị lớn ở Việt Nam, ô nhiễm không khí ảnh hưởng tới hoạt động của người dân mọi lúc, mọi nơi, nhất là thủ đô Hà Nội. Đây là một trong những thành phố ô nhiễm nhất châu Á. Mức độ ô nhiễm của Hà Nội tương đương thành phố Dehil và Karachi, hai trong 10 thành phố ô nhiễm không khí nhất thế giới. Nếu không có biện pháp giảm thiểu, nồng độ bụi ở Hà Nội sẽ tăng lên tới $200 \mathrm{mg} / \mathrm{m}^{3}$, gấp 10 lần mức khuyến cáo của Tổ chức $Y$ tế thế giới. Nếu chất lượng không khí ở Hà Nội và các thành phố lớn ở Việt Nam tiếp tục đi xuống, các trường hợp nhiễm bệnh do ô nhiễm không khí sẽ tăng gấp đôi vào năm 2020". Còn theo số liệu của Trung tâm Quan trắc môi trường, Tổng cục Môi trường Việt Nam cho thấy tại nhiều nút giao thông như Kim Liên- Giải Phóng, Phùng Hưng - Hà Đông, những khu vực đông dân cư, nồng độ bụi thường cao hơn mức cho phép, có lúc lên gấp 7 lần. Các khí ô nhiễm khác như $\mathrm{C} 0$, $\mathrm{SO}_{2}$ đang có xu hướng tăng.

\section{Dũ̃ liệu sử dụng}

Vệ tinh LANDSAT 8 OLI được phóng thành công vào ngày 11 tháng 2 năm 2013 , có hai bộ cảm biến chính: bộ thu nhận ảnh mặt đất (OLI - Operational Land Imager) và bộ cảm biến hồng ngoại nhiệt (TIRS Thermal Infrared Sensor). (xem bảng 1)

- Ảnh Landsat 8 OLI sử dụng trong nghiên cứu này là cảnh ảnh có số hiệu: LC81270452013352LGN00 chụp ngày 18 tháng 12 năm 2013 và được tải xuống từ trang http://landsat.usgs.gov.

\section{Phương pháp nghiên cứu}

Quá trình nghiên cứu được chia thành các bước sau: Thu thập dữ liệu, tiền xử lý ảnh, xử lý dữ liệu và đánh giá các kết quả. Tất cả các bước tiền xử lý và xử lý dữ liệu được thực hiện bằng cách sử dụng các phần mềm MathLap, ERDAS image.

\subsection{Tiền xử lý ảnh}

Việc tiền xử lý ảnh được tiến hành bằng cách chuyển các giá trị số (DN - Digital Nember) sang giá trị bước xạ phổ hoặc phản xạ phổ. Có nhiều mức hiệu chỉnh bức xạ. Đầu tiên chuyển đổi $\mathrm{DN}$ thành giá trị bức xạ tại đầu thu, thứ hai là chuyển đổi bức xạ phổ tại đầu thu về bức xạ phổ ở bề mặt trái đất, cuối cùng tiến hành hiệu chỉnh khí quyển ảnh để loại bỏ ảnh hưởng của điều kiện khí quyển đển chất lượng ảnh.

\subsubsection{Chuyển đổi $D N$ sang giá trị bức xạ phổ tại đỉnh khí quyển (TOA)}

Dữ liệu ảnh Landsat 8 OLI được chuyển đổi sang dữ liệu bức xạ phổ đỉnh khí quyển sử dụng công thức sau:

$$
L_{\lambda}=M_{L} * Q_{c a l}+A_{L}
$$

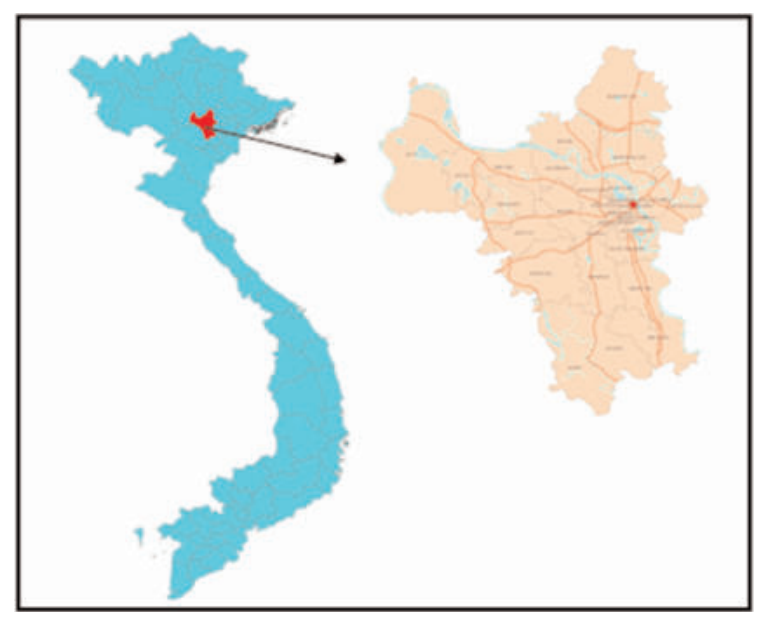

Hình 1: Khu vực nghiên cứu 
Bảng 1: Các kênh ảnh và độ phân giải không gian của ảnh Landsat 8 OLI

\begin{tabular}{|l|c|c|}
\hline \multicolumn{1}{|c|}{ Kênh ảnh } & Bước sóng $(\boldsymbol{\mu m})$ & Độ phân giải $(\mathbf{m})$ \\
\hline Kênh 1 - Coastal aerosol & $0.43-0.45$ & 30 \\
\hline Kênh 2 - Blue & $0.45-0.51$ & 30 \\
\hline Kênh 3 - Green & $0.53-0.59$ & 30 \\
\hline Kênh 4 - Red & $0.64-0.67$ & 30 \\
\hline Kênh 5 - Near Infrared (NIR) & $0.85-0.88$ & 30 \\
\hline Kênh 6 - SWIR 1 & $1.57-1.65$ & 30 \\
\hline Kênh 7 - SWIR 1 & $2.11-2.29$ & 30 \\
\hline Kênh 8 - Panchromatic & $0.50-0.68$ & 15 \\
\hline Kênh 9 - Cirrus & $1.36-1.38$ & 30 \\
\hline Kênh 10 - Thermal Infrared (TIRS) 1 & $10.60-11.19$ & 100 \\
\hline Kênh 10 - Thermal Infrared (TIRS) 2 & $11.50-12.51$ & 100 \\
\hline
\end{tabular}

Trong đó:

$L_{\lambda}$ - Bức xạ phổ đỉnh khí quyển (Watts/( $\left.\mathrm{m}^{2 *} \operatorname{srad}^{*} \mu \mathrm{m}\right)$ )

$\mathrm{M}_{\mathrm{L}}$ - Hệ số thay đổi tỷ lệ bức xạ của kênh ảnh theo tính chất đa bội, được lấy trong tệp dữ liệu metadata (RADIANCE_MULT_BAND_x, trong đó $x$ là kênh ảnh)

$A_{L}$ - Hệ số thay đổi tỷ lệ bức xạ của kênh ảnh theo tính chất cộng dồn, được lấy trong tệp dũ liệu metadata (RADIANCE_ADD_BAND_x, trong đó $x$ là kênh ảnh)

$Q_{c a l}$ - Lượng tử hóa và hiệu chuẩn tiêu chuẩn giá trị số của kênh ảnh (DN)

\subsubsection{Chuyển đổi giá trị số $D N$ sang phản xạ tại đỉnh khí quyển}

Dữ liệu các kênh ảnh Landsat 8 OLI chuyển đổi thành phản xạ tại đỉnh khí quyển TOA bằng cách sử dụng hệ số phản xạ hồi quy được cung cấp trong tệp dữ liệu matadata (tệp tin MTL). Phương trình sau đây được sử dụng để chuyển đổi các giá trị $D N$ sang phản xạ TOA đối với dữ liệu Landsat 8 OLI như sau:

$$
\rho \lambda^{\prime}=M_{\rho} * Q_{\text {cal }}+A_{\rho}
$$

Trong đó:

$\rho \lambda^{\prime}$ - TOA Phản xạ tại đỉnh khí quyển, chưa hiệu chỉnh góc tới

$\mathrm{M}_{\rho}$ - Hệ số thay đổi tỷ lệ phản xạ của kênh ảnh theo tính chất đa bội, được lấy trong tệp dữ liệu metadata (REFLECTANCE_MULT_BAND_x, trong đó $x$ là kênh ảnh)

$A_{\rho}$ - Hệ số thay đổi tỷ lệ phản xạ của kênh ảnh theo tính chất cộng dồn, được lấy trong tệp dữ liệu metadata (REFLECTANCE_ADD_BAND_x, trong đó $x$ là kênh ảnh)

$Q_{c a l}$ - Lượng tử hóa và hiệu chuẩn tiêu chuẩn giá trị số của kênh ảnh (DN)

TOA phản xạ đỉnh khí quyển khi hiệu chỉnh góc tới mặt trời:

\section{Trong đó:}

$$
\rho \lambda=\frac{\rho \lambda^{\prime}}{\cos \left(\theta_{S 2}\right)}=\frac{\rho \lambda^{\prime}}{\sin \left(\theta_{S E}\right)}
$$

$\lambda$ - TOA Phản xạ tại đỉnh khí quyển $\theta_{S E}$ - Góc tới mặt trời (SUN_ELEVATION).

$\theta_{S Z}$ - Góc thiên đỉnh mặt trời;

$$
\theta_{S Z}=90^{\circ}-\theta_{S E}
$$

\subsubsection{Hiệu chỉnh khí quyển}

Ảnh Landsat 8 OLI được cung cấp cho 
phép chúng ta chuyển đổi trực tiếp từ giá trị số của ảnh (DN) sang phản xạ đỉnh khí quyển $(T O A)$. Tuy nhiên, cần phải hiệu chỉnh khí quyển khi xác định phản xạ tại bề mặt đất. Theo [4] phản xạ mặt đất $(\rho)$ được tính theo công thức sau:

$$
\rho=\frac{\pi *\left(L_{\lambda}-L_{p}\right) * d^{2}}{\left.T_{V} *\left\{\left(\operatorname{ESUN}_{\lambda} * \cos \theta_{S z} * T_{z}\right)+E_{\text {down }}\right)\right\}}
$$

Trong đó:

$L_{P}$ - Bức xạ đường truyền,

$T_{V}$ - Hàm truyền bức xạ qua khí quyển từ bề mặt trái đất về đầu thu,

$T_{Z}$ - Hàm truyền bức xạ qua khí quyển từ mặt trời về bề mặt quả đất,

$E_{\text {down }}$ - Bức xạ phổ đi tới mặt phẳng địa hình của đối tượng.

$E \operatorname{SUN}_{\lambda}$ - Bức xạ phổ mặt trời ở mặt phẳng địa hình vuông góc với tia sáng mặt trời

d - Khoảng cách từ trái đất đến mặt trời.

Để hiệu chỉnh khí quyển ở đây chúng tôi sử dụng phương pháp trừ đối tượng tối DOS (Dark Object Subtraction) của [2] và xác định bức xạ đường truyền theo [5]. Đối với ảnh Landsat 8 OLI chúng ta có công thức như sau:

$L_{p}=L_{\min }-0.01 * \frac{T_{V^{*}} *\left\{\left(\operatorname{ESUN}_{\lambda} * \cos \theta_{S 2} * T_{2}\right)+E_{\text {down }}\right\}}{\pi * d^{2}}$

Trong đó, $L_{\min }$ là giá trị bức xạ phổ bé nhất của kênh ảnh, được lấy trong tệp dữ liệu metadata (RADIANCE_MINIMUM_BAND_x, trong đó $x$ là kênh ảnh)

Với phương pháp DOS phụ thuộc vào việc xác định các thông số $T_{V}, T_{Z}$ và $E_{d o w n}$ mà chia ra các phương pháp khác nhau (DOS1, DOS2, DOS3, DOS4) có độ chính xác khác nhau .

Trong nghiên cứu này, chúng tôi sử dụng DOS1, trong đó các thông số được xác định theo [4]: $T_{V}=1 ; T_{Z}=1 ; E_{\text {down }}=0$. Lúc này bức xạ đường truyền được tính theo công thức:

$$
L_{p}=L_{\min }-0.01 * \frac{\operatorname{ESUN}_{\lambda} * \cos \theta_{S Z}}{\pi * d^{2}}
$$

Và phản xạ mặt đất được tính theo công thức sau:

$$
\rho=\frac{\pi *\left(L_{\lambda}-L_{P}\right) * d^{2}}{\operatorname{ESUN}_{\lambda} * \cos \theta_{S z}}
$$

\subsection{Mối quan hệ giữa độ dày sol khí (AOT) và hàm lượng bụi PM10}

Năng lượng mặt trời đi vào lớp Sol khí ở tầng đối lưu, dưới tác động của các phân tử khí ô nhiễm và các hạt bụi, một phần bị phản xạ ngay trong lớp Sol khí rồi đi về đầu thu ảnh của vệ tinh, một phần tia nắng đi tới đối tượng trên bề mặt đất rồi phản xạ đi về đầu thu ảnh của vệ tinh. Dựa trên sự suy giảm năng lượng tới đầu thu vệ tinh do bị hấp thụ, tán xạ của các phân tử khí ô nhiễm và các hạt bụi từ đấy tính toán hàm lượng bụi trong không khí.

Sau khi hiệu chỉnh khí quyển, ta tính được phản xạ ở đỉnh của khí quyển (TOA) và phản xa mặt đất từ đó ta tính được phản xạ khí quyển. Từ đó, tính độ dày sol khí (AOT) như sau được đưa ra bởi [8]

$$
\operatorname{AOT}(\lambda)=a_{0} R(\lambda)
$$

Với:

$$
\begin{gathered}
\mathrm{R}(\lambda)=\rho_{\mathrm{a}}\left(\theta_{\mathrm{sz}}, \theta_{\mathrm{v}}, \phi\right) \\
\mathrm{a}_{\mathrm{o}}=\left(\frac{4 \mu \mu_{\circ}}{\omega_{\mathrm{o}} \mathrm{P}_{\mathrm{a}}\left(\theta_{\mathrm{sz}}, \theta_{\mathrm{v}}, \phi\right)}\right)
\end{gathered}
$$

Trong đó:

$\mathrm{R}(\lambda)$ - Hàm phản xạ khí quyển tương ứng với bức sóng $(\lambda)$

$P_{\mathrm{a}}\left(\theta_{S Z}, \theta_{\mathrm{v}}, \phi\right)$ - Hàm tán xạ Sol khí

$\theta_{S Z}$ - Góc thiên đỉnh mặt trời

$\theta_{v}-$ Phương vị góc nhìn

$\phi$ - Góc phương vị tương đối

$\mu$ - Cosin của góc nhìn

$\mu_{\mathrm{o}}$ - Cosin của hướng chiếu sáng 


\section{$\omega_{0}$ - Hệ số tán xạ albedo}

Phương trình (8) được viết lại cho ba kênh ảnh như sau:

$$
\operatorname{AOT}(\lambda)=a_{0} R_{\lambda 1}+a_{j} R_{\lambda 2}+a_{2} R_{\lambda 3}
$$

Trong đó $\mathrm{R}_{\lambda \mathrm{i}}$ là phản xạ khí quyển $(\mathrm{i}=1$, 2 và 3 tương ứng với bước sóng vệ tinh), và $\mathrm{a}_{\mathrm{j}}$ là hệ số thuật toán $(\mathrm{j}=0,1$ và 2$)$ được xác định bằng thực nghiệm.

Mối quan hệ giữa $\mathrm{PM}$ và $\mathrm{AOT}$ bắt nguồn từ một lớp khí quyển đồng nhất đơn thuần chứa các hạt Sol khí hình cầu. Nồng độ tập trung ở bề mặt thu được sau khi sấy mẫu không khí được đưa ra bởi [6].

$$
\mathrm{PM}_{\mathrm{X}}=\frac{4}{3} \pi \rho \int_{0}^{\mathrm{X} / 2} \mathrm{r}^{3} \mathrm{n}(\mathrm{r}) \mathrm{dr}
$$

Trong đó $n(r)$ mô tả sự phân bố kích thước hạt trong điều kiện khô và $\rho$ là mật độ khối lượng Sol khí

Do đó, ta thấy rằng rằng hàm lượng $\mathrm{PM}$ tương quan tốt hơn với $A O T$ trực tiếp. Bằng cách thay thế $\mathrm{AOT}$ bằng $\mathrm{PM} 10$ vào phương trình (9) ta có phương trình (11), và thuật toán cho kênh ảnh hoặc bước sóng $(\lambda)$, PM10 được đơn giản hóa bởi $[7,8]$

$$
\text { PM10 }=a_{0} R_{\lambda 1}+a_{j} R_{\lambda 2}+a_{2} R_{\lambda 3}
$$

Trong đó $R_{\lambda i}$ là phản xạ khí quyển ( $i=1$, 2 và 3 tương ứng với bước sóng vệ tinh), và $a_{i}$ là hệ số thuật toán ( $j=0,1$ và 2$)$ được xác định bằng thực nghiệm

Sau khí tính phản xạ khí quyển và số liệu đo PM10 thực địa, sử dụng phân tích thuật toán hồi quy để xây dựng các mô hình tính toán PM10. Mô hình tính PM10 được lựa chọn dựa trên việc so sánh các giá trị hệ số tương quan $R$ và sai số RMSE thấp nhất, trong đó giá trị hệ số tương quan cao nhất, $\mathrm{R}$ là 0.888 và mô hình hồi quy tuyến tính như sau được cung cấp bởi [8].

$$
P M 10=396 R_{\lambda 1}+253 R_{\lambda 2}-194 R_{\lambda 3}
$$

Trong đó $\mathrm{PM} 10$ là nồng độ $\mathrm{PM} 10$ có đơn vị $\left(\mu \mathrm{g} / \mathrm{m}^{3}\right), \mathrm{R}_{\lambda 1}, \mathrm{R}_{\lambda 2}$ và $\mathrm{R}_{\lambda 3}$ giá trị phản xạ khí quyển ứng với các kênh phổ xanh (blue), xanh lá (green) và đỏ (red), tương ứng với các kênh 2,3 và 4 của ảnh Landsat $8 \mathrm{OLI}$

\section{Kết quả thực nghiệm}

Sử dụng các phần mềm MathLap, ERDAS, ArcGIS và so sánh chỉ số AQI (chỉ số chất lượng không khí), chúng ta có bản đồ PM10 thể hiện (xem hình 2, hình 3).

Từ bản đồ so sánh với thực tế và các báo cáo về ô nhiễm không khí của thành phố. Ta thấy mật độ PM10 phản ánh đúng thực tế, ô nhiễm PM10 tập trung ở các khu đô thị đang được xây dựng và các tuyến giao thông lớn.

\section{Kết luận}

Kết quả của nghiên cứu này chỉ ra rằng ô nhiễm không khí có thể được xác định bằng cách sử dụng dữ liệu ảnh vệ tỉnh Landsat $8 \mathrm{OLI}$, với ưu điểm có thể xác định trên một diện rộng lớn.

Hình ảnh Landsat $8 \mathrm{OLI}$ đã được sử dụng thành công để tính nồng độ PM10 trên Thành phố Hà Nội. Việc tính toán PM10 dựa trên mô hình phản xạ phổ, bản đồ bụi phản ảnh thực tế các khu vực tập trung ô nhiễm bụi của Thành phố Hà Nội. Kết quả cho thấy rằng ô nhiễm không khí $\mathrm{PM} 10$ có thể được tính toán bằng cách sử dụng giá trị phản xạ của các kênh ảnh Landsat 8 OLI.

Tuy nhiên, việc sử dụng mô hình tính PM10 của khu vực này cho khu vực khác ảnh hưởng đến tính chính xác của các kết quả tính toán. Nghiên cứu trong tương lai cần xem xét sử dụng nhiều trạm ô nhiễm không khí và các dữ liệu đo thực địa, sau đó tính toán một mô hình hồi quy để xác định PM10 sử dụng ảnh Landsat 8 OLI cho khu vực cần nghiên cứu. Cũng như phương pháp điều chỉnh khí quyển của Landsat 8 OLI để đạt được độ chính xác tốt hơn và đáng tin cậy hơn. $\mathrm{O}$

\section{Tài liệu tham khảo}

[1]. Asmala Ahmad and Mazlan Hashim, 


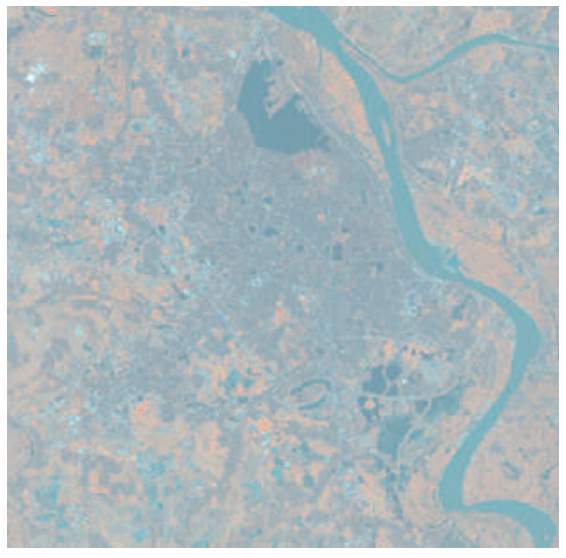

Hình 2: Ảnh Landsat 8 OLI Hà Nội

(2002). Determination of haze using NOAA14 AVHRR satellite data, [Online] available: http://www.gisdevelopment.net/aars/acrs/20 02/czm/050.pdf.

[2]. Chavez, P.S., 1996. Image - based atmostpheric correction - revisited and improved. Photogrammetric Engineering and Remote Sensing, Vol 62, 1025-1036 sensing. [Falls Church, Va.] American Society of Photogrammetry, 62, 1025-1036.

[3].Finn, M.P., Reed, M.D., and Yamamot o, K.H., 2012. A Straight Forward Guide for Processing Radiance and Reflectance for EO-1 ALI, Landsat 5 TM, Landsat 7 ETM+, and ASTER. Unpublished Report from USGS/Center of Excellence for Geospatial Information Science, $8 p$, http://cegis.usgs.gov.

[4]. Moran, M., Jackson, R., Slater. P., and Teillet, P., 1992. Evaluation of simplified procedures for retrieval of Land surface reflectance factors from satellite sensor output. Remote Sensing of Environment, 41, 169-184.

[5]. Sobrino, J., Jiménez- Muñoz, J. C., and Paolini, L., 2004. Land surface temperature retrieval from LANDSAT TM 5 . Remote Sensing of Environment, Elsevier, 90, 434-440.

[6]. Koelemeijer, R. B. A., Homan, C. D.,

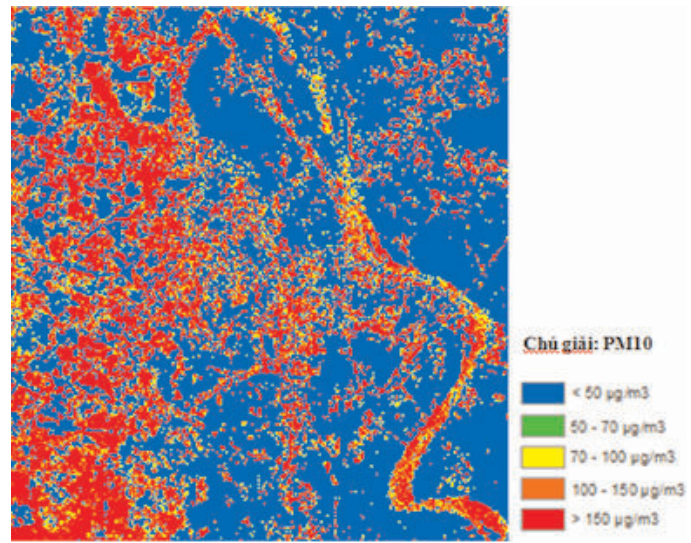

Hình 3: Bản đồ bụi PM10 Hà Nội

\& Matthijsen, J., 2006. Comparison of spatial and temporal variations of aerosol optical thickness and particulate matter over Europe. Atmospheric Environment, 40, 5304-5315.

[7]. Lim HS, MatJafri MZ, Abdulla K, Mohd NS, Sultan AS (2004) Remote Sensing of PM10 From Landsat TM Imagery. 25th ACRS 2004 Chiang Mai, Thailand.

[8]. Nadzri, O., Mohd, Z.M.J., Lim, H.S., 2010. Estimating Particulate Matter Concentration over Arid Region Using Satellite Remote Sensing: A Case Study in Makkah, Saudi Arabia. Modern applied Science 4: 131-142.

[9]. Sifakis, N. \& Deschamps, P.Y. (1992). Mapping of air pollution using SPOT satellite data, Photogrammetric Engineering \& Remote Sensing, 58(10), 1433 - 1437.

[10]. Ung, A., Weber, C., Perron, G., Hirsch, J., Kleinpeter, J., Wald, L. and Ranchin, T., 2001a. Air Pollution Mapping Over A City - Virtual Stations And Morphological Indicators. Proceedings of 10th International Symposium "Transport and Air Pollution" September 17 - 19, 2001 Boulder, Colorado USA.

[11]. Ung, A., Wald, L., Ranchin, T., Weber, C., Hirsch, J., Perron, G. and Kleinpeter, J., 2001b. , Satellite data for Air 
Pollution Mapping Over A City- Virtual Stations, Proceeding of the 21th EARSeL Symposium, Observing Our Environment From Space: New Solutions For A New Millenium, Paris, France, 14 - 16 May 2001, Gerard Begni Editor, A., A., Balkema, Lisse, Abingdon, Exton (PA), Tokyo, pp. 147-151.

[12]. Weber, C., Hirsch, J., Perron, G., Kleinpeter, J., Ranchin, T., Ung, A. and Wald, L. 2001. Urban Morphology, Remote Sensing and Pollutants Distribution: An Application To The City of Strasbourg, France. International Union of Air Pollution Prevention and Environmental Protection Associations (IUAPPA) Symposium and Korean Society for Atmospheric Environment (KOSAE) Symposium, 12th World Clean Air \& Environment Congress, Greening the New Millennium, 26 - 31 August 2001, Seoul, Korea.

[13]. J.-C. Péré, V. Pont, M. Mallet, B. Bessagnet, 2009. Mapping of PM10 surface concentrations derived from satellite observations of aerosol optical thickness over South-Eastern France. Atmospheric Research 91 (2009) 1-8.

[14]. Luong Chinh Ke, Ho Thi Van Trang, Tran Ngoc Tuong, Nguyen Le đang, 2010. Detecting Air Pollution In Vietnam By Optical Satellite Images, E-proceedings of The 31th Asian Conference on Remote Sensing
(ACRS2010), 1-5 November 2010, Hanoi, Vietnam.

[15]. Trần Thị Vân, Trịnh Thị Bình, Hà Dương Xuân Bảo, 2012. Nghiên cứu khả năng phát hiện ô nhiễm bụi trên khu vực đô thị bằng công nghệ viễn thám nhằm hỗ trợ quan trắc môi trường không khí, Tạp chí phát triển khoa học và công nghệ, Tập 16, Số M2-2012.

[16]. Trần Thị Vân, Nguyễn Phú Khánh, Hà Dương Xuân Bảo, 2014. Viễn thám độ dày quang học mô phỏng phân bố bụi PM10 khu vực nội thành Thành phố Hồ Chí Minh, Tạp chí phát triển khoa học ĐHQGHN: Các Khoa học Trái đất và Môi trường, tập 30 , Số 2 (2014) 52-62.

[17]. Trần Xuân Trường, Vương Trọng Kha, Nguyễn Văn Mạnh 2013, Nghiên cứu xây dựng chương trình giám sát ô nhiễm không khí vùng mỏ từ dữ liệu ảnh vệ tinh, Tạp chí Công ngiệp Mỏ, số 26 - 2013.

[18]. Bộ Tài nguyên và Môi trường, Báo cáo môi trường quốc gia 2013- Môi trường không khí, Số ĐKKHXB: 31-2014/CXB/19915/BĐ; Quyết định số 51/QĐXB ngày 01 tháng 08 năm 2014, ISBN: 978-604-904248-5.

[19].

https://landsat.usgs.gov/Landsat8_Using_P roduct.php. $O$

\section{Summary}

\section{Study the ability to detect air pollution PM10 from data satellite Landsat 8 OLI image over Hanoi city}

\section{Nguyen Nhu Hung, Military Technical Academy}

\section{Tran Van Anh, Hanoi University of Mining and Geology}

In this study we research about the potentialy of retrieving concentrations of particulate matter with diameters less than ten micrometer (PM10) in the atmosphere using the Landsat 8 satellite images over Hanoi city. The research focus on testing of process for air pollution determination, starting from the atmospheric correction of Landsat 8 by means of the DOS, calculated AOT, the relationship function between AOT and PM10, since then the model of PM10 can be calculated and selected from the Landsat $8 \mathrm{OLI}$ images in the central of Hanoi area. $\mathrm{O}$ 